\title{
Clinical similarities of hereditary progressive/dopa responsive dystonia caused by different types of mutations in the GTP cyclohydrolase I gene
}

Yoshiko Tamaru, Makito Hirano, Hidefumi Ito, Junichiro Kawamura, Sadayuki Matsumoto, Terukuni Imai, Satoshi Ueno

\begin{abstract}
Objective-Hereditary progressive dystonia with pronounced diurnal fluctuation ((HPD)/dopa responsive dystonia (DRD)) is a childhood onset dystonia which responds to levodopa. Various clinical signs and symptoms of HPD/DRD have been recognised to date. Mutations in the GTP cyclohydrolase I (GTP-CH-I) gene were recently identified as the cause of HPD/ DRD. In the present study, the GTP-CH-I gene and the clinical features of eight HPD/DRD patients from six families were analysed to determine the correlations between clinical expression and the mutations in the GTP-CH-I gene.
\end{abstract}

Methods-The exons, exon-intron junctions, and an indispensable part of the 5 , flanking region of the GTP-CH-I gene were sequenced in the eight clinically diagnosed patients with HPD/DRD and their asymptomatic parents.

Results-Three independent mutations in the GTP-CH-I gene were found in three patients. One of the patients and her asymptomatic mother were heterozygous for a novel mutation at the initiation codon. The three patients with dissimilar GTP-CH-I mutations exhibited similar clinical features. The other five patients with normal sequences presented several features not manifested by the three patients with the mutations. No mutation was found in the 5' flanking region of any patients or their parents.

Conclusions-A novel initiation codon mutation was found in a Japanese patient with HPD/DRD. The clinical manifestations common to the patients with HPD/ DRD with a mutated GTP-CH-I gene were also identified. Although focal manifestations of HPD/DRD associated with the mutations of this gene will be broadened, it is inferred that these clinical features are fundamental to HPD/DRD caused by mutations in this gene.

(F Neurol Neurosurg Psychiatry 1998;64:469-473)

Keywords: dystonia; GTP cyclohydrolase I; gene analysis

Correspondence to: Dr Satoshi Ueno, Department of Medical Genetics, Nara Medical

University, Kashihara, Nara, 634, Japan.

Received 7 January 1997 and in revised form 10 July 1997 Accepted 21 August 1997 features of HPD/DRD, including its autosomal dominant inheritance and the diurnal variation of symptoms aggravated toward the evening and alleviated in the morning after sleep. However, the genetic penetrance ratio of $\mathrm{HPD} /$ DRD seems to be low, and the degree of diurnal fluctuation varies from one patient to another. Besides these main features, variably concurrent symptoms such as hyperreflexia, tremor, and hypertonus resembling rigidity may develop in some patients with HPD/DRD. ${ }^{2}$ Nomura and Segawa ${ }^{4}$ reported that HPD/DRD can present intrafamilial and interfamilial variations with respect to symptoms, and that because of partial similarities of the manifestations with other movement disorders, some patients can be diagnosed as having a dystonic variety of juvenile parkinsonism ${ }^{5}$ or young age onset Parkinson's disease. ${ }^{6}$ By contrast, among the patients described as HPD there were some that should be considered as having other dystonic syndromes. ${ }^{4}$

Mutations in the GTP cyclohydrolase I (GTP-CH-I) gene were recently identified as the cause of HPD/DRD. ${ }^{7}$ Subsequent reports ${ }^{8-12}$ described several independent mutations of this gene in other patients with $\mathrm{HPD} / \mathrm{DRD}$. However, no alterations were detected in the coding region of the gene of certain patients clinically diagnosed with HPD/DRD. ${ }^{4}{ }^{10}$ Moreover, a point mutation in the tyrosine hydroxylase gene was described in one family with HPD/DRD. ${ }^{13}$ These diverse findings may be due, at least in part, to the inclusion criteria used for patients with HPD/ DRD, as there have been no definite criteria based on pathological and molecular features. Nevertheless, the previous findings would indicate that HPD/DRD is heterogeneous at the molecular level.

There have been a few reports that present adequate clinical information on genetically verified cases of HPD/DRD. Hence, for the further clarification of this syndrome, it is essential that the correlations between the clinical manifestations and molecular genetic features be determined. We considered this point in the present study and we report the results of a GTP-CH-I gene analysis of eight clinically diagnosed patients with HPD/DRD from six families. We found three independent mutations of the gene, including a novel mutation at the initiation codon. We compared the clinical features of the patients with HPD/ DRD with GTP-CH-I mutations with those of the patients without the mutations. 


\begin{tabular}{|c|c|c|c|c|c|c|c|c|}
\hline & \multicolumn{8}{|l|}{ Patient No } \\
\hline & 1 & 2 & 3 & 4 & 5 & 6 & 7 & 8 \\
\hline Age $(y) / \operatorname{Sex}$ & $36 / \mathrm{F}$ & $26 / \mathrm{F}$ & $23 / \mathrm{M}$ & $18 / \mathrm{F}$ & $23 / \mathrm{M}$ & $45 / \mathrm{F}$ & $58 / \mathrm{F}$ & $16 / \mathrm{M}$ \\
\hline Age at onset (y) & 8 & 7 & 10 & 2 & 2 & 8 & 8 & 8 \\
\hline Initial site of dystonia & Feet $(\mathrm{L}>\mathrm{R})$ & Feet $(\mathrm{L}>\mathrm{R})$, neck & $\mathrm{R}$ foot, neck & Feet, neck & All limbs & $\mathrm{L}$ arm & $\mathrm{R}$ foot & $\mathrm{R}$ foot \\
\hline Diurnal fluctuation & + & + & + & + & + & + & + & + \\
\hline Dopa responsiveness (mg/day) & +200 & +50 & +200 & +60 & +45 & $+600 \dagger$ & $+600 \dagger$ & $+800 \dagger$ \\
\hline \multicolumn{9}{|l|}{ Neurological signs: } \\
\hline Legs affected more than arms & + & + & + & - & - & - & + & + \\
\hline $\begin{array}{l}\text { GTP cyclohydrolase } 1 \text { gene } \\
\text { mutations }\end{array}$ & $\mathrm{ATG} \rightarrow \mathrm{ATC}$ & $\mathrm{A} \rightarrow \mathrm{C}$ (His 144Pro) & $\begin{array}{l}\text { Intron2, } \\
\mathrm{GT} \rightarrow \mathrm{CT}\end{array}$ & - & - & - & - & - \\
\hline Others & & & & Dysarthria & $\begin{array}{l}\text { Dysarthria, } \\
\mathrm{MR}^{\star}\end{array}$ & & $\begin{array}{l}\text { Spontaneous } \\
\text { remission }\end{array}$ & \\
\hline
\end{tabular}

$\star \mathrm{MR}=$ mental retardation

† Without decarboxylase inhibitor.

\section{Materials and methods}

\section{PATIENTS}

The table shows the clinical profiles of the eight patients from six independent Japanese families. Four cases were sporadic (patients 1, 2, 3, and 8) and four were familial, the offspring of two families (patients 4 to 7 ). Consanguinity was not documented in these patients.

All eight patients fulfilled the criteria for HPD/DRD described by Nygaard et $a l^{3}$ Briefly, the age at onset of clinical symptoms ranged from 2 to 10 years. The presenting symptom was focal postural dystonia that progressed gradually into generalised dystonia within several years. The clinical response to levodopa was definite and without a side effect such as motor response fluctuations or dopa induced dyskinesia. Diurnal fluctuations of symptoms were evident, but their range varied from one patient to another. Cranial CT or MRI disclosed no abnormalities.

\section{Case reports}

PATIENT 1

A 36 year old woman had had gait difficulties due to intermittent internal rotation of her legs, particularly the left one, since the age of eight. The symptoms were aggravated towards evening and gradually spread to the other limbs. Postural tremor became apparent after the age of 20 . During her 30 s, she could walk for one hour in the morning, becoming immobile towards evening. On examination at the age of 35 , both feet tended to turn inward and plantarflex. She walked with her left foot circumflexed. Dystonic hypertonicity was noted at the left ankle and there was functional limitation of both ankles. The deep tendon reflexes were normal and plantar responses were flexor. She had a postural tremor in her hands. Small doses of levodopa (200 mg/day, with decarboxylase inhibitor) provided a considerable and sustained improvement of her symptoms.

PATIENT 2

At the age of 7 , this 26 year old woman developed gait disturbances due to pes equinovarus of both legs, dominantly in the left one. Her head turned involuntarily to the right, which worsened in the afternoon. At the age of 10, the patient underwent surgery to lengthen both Achilles tendons, but without appreciable benefit. The symptoms progressed slowly over the next eight years. When she was 18 years old, she noticed clumsiness of her hands due to postural tremor. When admitted at age 18, the patient showed flexion-inversion of the feet that was more pronounced on the left, palmar flexion of the left hand, and torticollis to the right. She exhibited predominant pes equinovarus of the left side while walking, and intermittent dystonic posture of the left arm. The deep tendon reflexes were normal. Her symptoms have been completely controlled after she started levodopa therapy $(50 \mathrm{mg} /$ day, with decarboxylase inhibitor).

\section{PATIENT 3}

At the age of 10 , this 23 year old man noticed flexion-inversion of the right foot, especially in the afternoon. His dystonia gradually worsened and involved all limbs within five years; it was more pronounced in the lower limbs. On examination at the age of 22, the patient exhibited rigidity of the right elbow. $\mathrm{He}$ had pronounced dystonic posturing in the left hand and leg as well as scoliosis. He walked on his toes with torticollis to the left and carried his left arm in the flexed position. The deep tendon reflexes were normal and plantar responses were flexor bilaterally. After beginning levodopa therapy (200 mg/day, with decarboxylase inhibitor) his symptoms improved remarkably.

\section{PATIENTS 4 AND 5}

These patients ${ }^{14}$ were siblings. Patient 4 was an 18 year old woman. Her parents noticed that she developed mild torticollis and plantar flexion of both ankles when she was two years old. At the age of 4, walking became difficult in the evening with dystonic posture of the limbs and frequent falls. Examination at the age of 8 disclosed bilateral pes equinovarus, unsustained ankle clonus, and hyperactive reflexes with flexor plantar responses. She had a dystonic posture in all limbs with generalised rigidity, being more pronounced in the left. Alternating movements of the forearms and foot tapping were slow. Her speech was slightly dysarthric.

Patient 5 was the older brother of patient 4 . He was a 23 year old man who began to walk at 19 months, but was persistently unsteady. In the next year, he started walking on tiptoe and showed an intermittent involuntary flexion of both elbows, wrists, and fingers. The patient was dysarthric in the evening. When examined at the age of 12, he manifested flexioninversion of the feet and palmar-flexion of the 

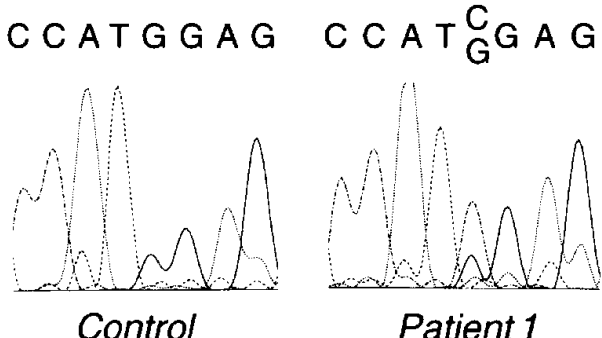

Patient 1

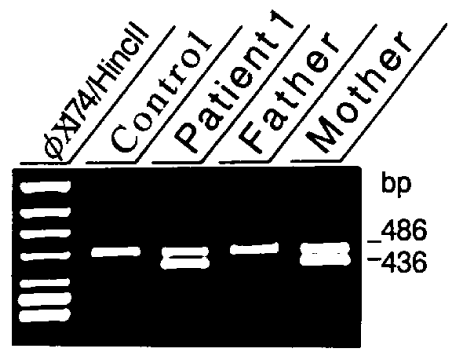

C

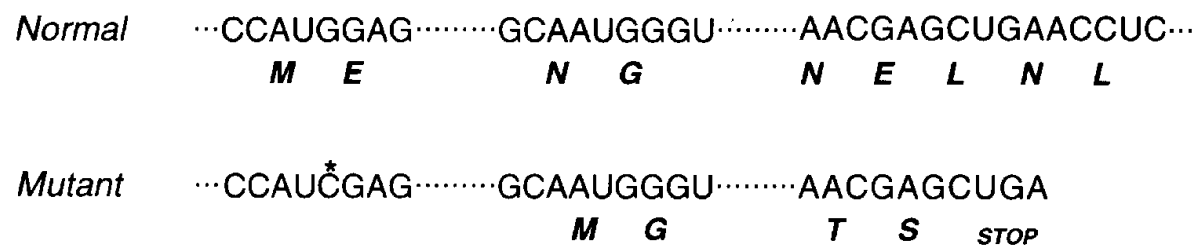

(A) Direct sequence analysis showing that the control subject is a homozygote of $G$ at the third nucleotide of the initiation codon ATG. Patient 1 is a heterozygote of $C$ and $G$ at the same position. (B) Identification of the initiation codon mutation by Taq I restriction analysis. Patient 1 and her mother had two extra bands (50 and $436 \mathrm{bp}$ ) in addition to normal bands $(16$ and $486 \mathrm{bp})$. The 16 and $50 \mathrm{bp}$ bands are not visible in the gel. (C) The upper $m R N A$ sequence encodes normal GTP cyclohydrolase I, and lower sequence, the mutant one. ${ }^{\star}$ The $G$ to $C$ mutation at the third letter of the initiation codon. If the next outframe AUG is used as the initiation codon, the resulting product encodes a non-functional 46 amino acid peptide.

Sequences are based on the published data of Togari et al. ${ }^{17}$

wrists while walking. He also showed rigidity of the right elbow, hyperreflexia in all limbs, and unsustained ankle clonus without extensor plantar responses. Psychometric examination using the Suzuki-Binet intelligence scale disclosed a total IQ of 70; the principal disturbances were in verbal memory, calculation, and comprehension, which were not considered to be affected by his motor disability. The two siblings responded to levodopa with a considerable beneficial effect. Although there was slight residual dystonia, the improvements of their conditions have been maintained for 11 years.

PATIENTS 6 AND 7

These two patients were siblings. ${ }^{15}$ Patient 6 was a 45 year old woman. She was healthy until the age of 8 , when she experienced intermittent stiffness in her left arm. She could not run well as a student. At the age of 22, she developed difficulties in using her left hand. In her $40 \mathrm{~s}$, she was almost unable to move her left arm, and sometimes experienced gait difficulties. The patient's symptoms tended to become worse during the day, and she noticed that sleeping brought improvement. When examined at the age of 37, she exhibited dystonic posture of the left arm and torticollis to the left. She had slight rigidity of the left wrist and legs, and hyperreflexia without extensor plantar responses.

Patient 7 was the older sister of patient 6 . She was a 58 year old woman who at the age of 8 started walking on her right toe, particularly towards evening. Her dystonic posturing spread to her other limbs within three years. She noted a pronounced diurnal variation of her symptoms, with improvements after a short sleep. At the age of 23, the patient's symptoms spontaneously began to gradually ameliorate, and within the next six years her daily activities were no longer affected by dystonia, even in the evening. However, when she was 42 years old, the dystonia of both feet reappeared, involving all limbs within five years. Examination at the age of 50 disclosed bilateral pes equinovarus and dystonic posturing of the left hand and neck when walking. She exhibited rigid hypertonicity with normal deep tendon reflexes. The symptoms of both sisters have been totally controlled since beginning levodopa treatment (600 mg/day, without decarboxylase inhibitor). Their father had symptoms similar to those of patient 7.

\section{PATIENT 8}

A 16 year old boy started dragging his right leg and had occasional falls at the age of 9 . Within one year, he began walking in a stooped manner; his postural dystonia progressed to the left foot and right hand, which worsened in the afternoon. A neurological examination at the age of 11 showed postural tremor of both hands, normal deep tendon reflexes, Myerson's sign, and dystonic posturing of the lower limbs and right arm. Alternating movements of the upper limbs were slower on the right side. He walked with flexion of his right arm, bending his trunk forward. He was treated with levodopa (800 $\mathrm{mg} /$ day, without decarboxylase inhibitor), and almost all of his symptoms disappeared.

SEQUENCE AND RESTRICTION SITE ANALYSIS OF THE GTP-CH-I GENE

Genomic DNAs were extracted from peripheral blood leucocytes of the eight patients, their parents, and 50 unrelated normal control subjects. All exons and exon-intron junctions of the GTP-CH-I gene were amplified by polymerase chain reaction (PCR) and sequenced as previously described. ${ }^{8}$

To verify the sequencing results of the genomic DNA of patient 1 and to confirm the 
genotype of the parents and control subjects, we analysed the Taq I restriction sites of the genomic DNA. The PCR amplified fragments containing exon 1 of the GTP-CH-I gene were digested with Taq I at $65^{\circ} \mathrm{C}$ for three hours, electrophoresed in a $2.5 \%$ agarose gel, and stained with ethidium bromide.

We also amplified and sequenced the 5' flanking regions of exon $1,{ }^{16}{ }^{17} 588$ base pairs upstream from the first exon, using two primer pairs: 5'-TAAGAGTTGGTTCCTAATC GGT-3' and 5'-AGAGGCCTCGGCGCTG GGGA-3'， 5'-CCTGCGCTTGCGAACCC CTC-3' and 5'-TCTTCCCGGGACACGC CCGTGG-3'.

In addition, the fragment that encodes exon 11 of the tyrosine hydroxylase gene from eight patients was amplified by PCR using the primers described by Lüdecke et $a l,{ }^{13}$ and sequenced.

\section{Results}

Patient 1 and her asymptomatic mother were heterozygous for a $\mathrm{G}$ to $\mathrm{C}$ substitution in the initiation codon of the GTP-CH-I gene (figure A). This mutation results in the generation of a Taq I restriction site. The figure $\mathrm{B}$, shows that patient 1 and her mother had two extra restriction fragments ( 50 and $436 \mathrm{bp}$ ) in addition to the normal 16 and $486 \mathrm{bp}$ fragments. These restriction patterns confirmed that both were heterozygous for the mutation. No such restriction pattern was present in the father or the 50 unrelated normal controls.

Patient 2 and her asymptomatic father were heterozygous for a His to Pro substitution at codon 144 of the GTP-CH-I gene. ${ }^{12}$ Patient 3 and his healthy mother were heterozygous for a $\mathrm{G}$ to $\mathrm{C}$ transversion at the $5^{\prime}$ end of intron 2, causing the skipping of exon 1 to exon $3 .^{8}$ No mutations were found in the other two families (patients 4 and 5 and patients 6 and 7) and in one sporadic case (patient 8). We did not detect any mutations of the 5 ' flanking region of exon 1 in any of the eight patients and their asymptomatic parents. In addition, none of the patients with HPD/DRD had the Gln381Lys mutation in the tyrosine hydroxylase gene.

The table shows that the clinical manifestations of the three patients with HPD/DRD with mutations in the GTP-CH-I gene (patients 1-3) were similar. The initial symptom was gait disturbance due to unilaterally predominant foot dystonia. Within a few years the dystonia progressed and involved all four limbs. The legs were affected more than the arms, and the asymmetry of symptoms was preserved. A diurnal fluctuation of symptoms was evident, although its range varied from one patient to another. Small doses of levodopa controlled the symptoms permanently without any adverse effect such as motor response fluctuations or dopa induced dyskinesia.

By contrast, the other five patients (patients 4 to 8) who had normal sequences in the coding and 5' flanking regions of the GTP-CH-I gene presented several features not seen in the three patients with mutations. Patients 4 and 5 did not display an apparent asymmetry of symptoms; the upper limbs were involved to the same degree as the lower limbs, as was their articula- tion. The intellect of patient 5 was slightly impaired without any evidence of a neonatal insult. The dystonia of patient 6 started in an arm, and later the arms were more affected than the legs. The clinical courses of patient 7 and her father were unique with respect to the spontaneous remission of symptoms, which in patient 7 lasted for 19 years. Patient 8 showed dystonia of the trunk, predominantly when walking, and Myerson's sign within one year of onset.

\section{Discussion}

Patient 1 is the first reported patient with HPD/DRD associated with a novel initiation codon mutation ( $\mathrm{G}$ to $\mathrm{C}$ ) in the GTP-CH-I gene, which abolishes the first AUG codon. The next AUG codon lies at the position corresponding to amino acid number 20, resulting in a frame shift and a UGA termination codon located 139 nucleotides downstream. The putative translation product is a 46 amino acid peptide that is completely different from the normal GTP-CH-I (figure C). This base change in patient 1 was unlikely to be a benign polymorphism, as it was not present on 100 chromosomes from unrelated normal controls. Mutations in the initiation codon have been reported in various hereditary diseases, including $\beta$-thalassaemia ${ }^{18}$ and phenylketonuria. ${ }^{19}$ Ichinose $e t a l^{7}$ described two patients (family $\mathrm{Sa}$ ) associated with a frame shift mutation in the GTP-CH-I gene. The two base insertion mutation (ATGGAG to ATGGGGAG) was immediately after the initiation codon, resulting in a frame shift after the starting methionine. The GTP-CH-I activity of the phytohaemagglutinin stimulated mononuclear cells of these patients was less than $20 \%$ of the normal level. We thus speculate that the novel mutation in patient 1 causes a decrease in enzyme activity that leads to the phenotypic expression of HPD/DRD.

In patients 2 and $3,{ }^{812}$ we suggest that a dominant negative effect may explain the low penetrance. However, in patient 1, a dominant negative effect is not an acceptable explanation as the resulting mutant peptide would be unable to interact with the wild type GTP$\mathrm{CH}-\mathrm{I}$. Possible explanations for such cases include other regulatory gene(s) or environmental factors that may be involved in the manifestation of clinical symptoms. ${ }^{7}$ To test for the presence of one of such possible regulatory factors, we amplified and sequenced the indispensable part of the 5' flanking region of exon 1 of the GTP-CH-I gene. Because Witter et al have shown that the 452 base pair fragment from the 3 ' end of the promotor region had the normal promotor activity, ${ }^{22}$ we sequenced 588 bp upstream from the first exon. However, we found no mutations in this part of the 5' flanking region of any of the three patients with a mutated GTP-CH-I gene and their asymptomatic parents. Further detailed analyses of the whole promotor region are required.

It is of particular interest that the clinical manifestations of the patients with a mutant GTP-CH-I gene were essentially similar, although the underlying molecular alterations differed significantly in terms of the sites of the 
gene and of the encoded products. Furukawa et al presented the clinical information of three patients with HPD/DRD with a mutated GTP$\mathrm{CH}-\mathrm{I}$ gene. ${ }^{11}$ Although none of the mutations of their patients was at the same site as those identified in the present study, their patients' symptoms resembled those of our patients; the childhood onset dystonia started predominantly in the unilateral lower limbs, with evident laterality and lower limb predominance throughout the clinical course, and there was a dramatic and sustained response to levodopa without any side effects. Except for exaggerated deep tendon reflexes, their two Japanese patients showed essentially similar manifestations ${ }^{11}$ as those of our patients. However, the relevance of hyperreflexia in patients with $\mathrm{HPD} / \mathrm{DRD}$ remains unknown. The plantar responses are always flexor, ${ }^{1-3}$ and the histological examination of a patient with HPD/DRD with a mutant GTP$\mathrm{CH}-\mathrm{I}$ gene failed to disclose any alterations in the corticospinal pathway. ${ }^{23}$ In a patient of English-Irish ancestry examined by Furukawa et $a l,{ }^{11}{ }^{23}$ the predominant side of the foot dystonia at the onset of symptoms was not apparent by the provided history, but a later neurological examination disclosed that she exhibited right side pes equinovarus while walking. ${ }^{11}{ }^{23}$ The typical diurnal fluctuation was not evident in this patient; her symptoms tended to be worse in the morning. ${ }^{123}$ Bandmann et al described two English siblings with HPD/DRD who had a Lys224Arg mutation in the GTP-CH-I gene, and whose symptoms resembled athetoid cerebral palsy. ${ }^{10}$ Nevertheless, it remains to be established whether the inconstant presentation of diurnal fluctuation or a phenotype distinct from dystonia is due to racial or environmental differences. It is likely, however, that with further genetic analyses, more atypical cases will be disclosed.

Lüdecke et al have identified a point mutation in the tyrosine hydroxylase gene of patients with recessively inherited disease, whose symptoms resembled those of HPD/DRD. ${ }^{13}$ This finding suggests that clinically diagnosed HPD/DRD may represent a heterogeneous entity caused by various genetic defects. Hence, it is relevant in this context that our data indicate that there was considerable homogeneity with respect to the clinical manifestations of our three patients with HPD/DRD with mutated GTP-CH-I genes and those recently described by Furukawa et al. ${ }^{11}$

Conversely, several discordant features were identified in the other five patients with normal sequences in the exons and exon-intron junctions and the essential part of the 5' flanking region of the GTP-CH-I gene. None the less, their clinical presentations have convinced us that the best conceivable diagnosis of their illness is HPD/DRD. Evidence has been obtained that certain patients with HPD/DRD do not have a mutation in the GTP-CH-I gene. ${ }^{7} \mathrm{~A}$ possible explanation for the disease in such patients includes a mutation that may lie outside the coding region of the gene or in other genes. ${ }^{7}$ Additional investigations of such cases may dis- close mutations in other genes related to $\mathrm{HPD} / \mathrm{DRD}$, or categorise them as belonging to entities that are distinct from this disorder. Further molecular genetic studies are required to determine the total expression of the mutant GTP-CH-I gene and to establish the clinical range of HPD/DRD with mutations in this gene.

$\mathrm{YT}$ and $\mathrm{MH}$ are visiting research fellows at Nara Medical University. This study was partly supported by a research grant from Nara Medical University, and by a Grant-in-Aid for Scientific Research from the Ministry of Education, Science and Culture of Japan. We thank Dr Koji Inui and Dr Toshihiko Suenaga for obtaining samples and medical histories from the patients. We thank Dr Fritz Herz for critical reading of the manuscript.

1 Segawa M, Hosaka A, Miyagawa F, et al. Hereditary progressive dystonia with marked diurnal fluctuation. In: Eldridge R, Fahn S, eds. Advances in neurology. New York: Raven Press, 1976;14:215-33.

2 Segawa M, Nomura Y. Hereditary progressive dystonia with marked diurnal fluctuation. In: Segawa M, ed. Hereditary progressive dystonia with marked diurnal fluctuation. New York: Parthenon, 1993;3-19.

3 Nygaard TG, Snow BJ, Fahn S, et al. Dopa-responsive dystonia: clinical characteristics and definition. In: Segawa $\mathrm{M}$, ed. Hereditary progressive dystonia with marked diurnal M, ed. Hereditary progressive dystonia with ma

4 Nomura Y, Segawa M. Intrafamilial and interfamilial variations of symptoms of Japanese hereditary progressive dystonia with marked diurnal fluctuation. In: Segawa $M$, ed. Hereditary progressive dystonia with marked diurnal fluctuation. New York: Parthenon, 1993;73-96.

5 Narabayashi H, Yokochi M, Iizaka R, et al. Juvenile parkinsonism. In: Vinken RJ, Bruyn GW, Klawans HL, eds. Handbook of clinical neurology. Amsterdam: Elsevier, $1986 ; 153$.

6 Quinn N, Critchley P, Marsden CD. Young onset Parkinson's disease. Mov Disord 1987;2:73-91.

7 Ichinose $\mathrm{H}$, Ohye T, Takahashi E, et al. Hereditary progressive dystonia with marked diurnal fluctuation caused by mutations in the GTP cyclohydrolase I gene. Nat Genet 1994;8:236-42.

8 Hirano M, Tamaru Y, Nagai Y, et al. Exon skipping caused by a base substitution at a splice site in the GTP cyclohydrolase I gene in a Japanese family with hereditary progressive dystonia/dopa responsive dystonia. Biochem progressive dystonia/dopa responsive
Biophys Res Commun 1995;213:645-51.

Biophys Res Commun 1995;213:645-51.
9 Ichinose H, Ohye T, Segawa M, et al. GTP cyclohydrolase I gene in hereditary progressive dystonia with marked diurnal fluctuation. Neurosci Lett 1995;196:5-8

10 Bandmann O, Nygaard TG, Surtees R, et al. Doparesponsive dystonia in British patients: new mutations of the GTP-cyclohydrolase I gene and evidence for genetic heterogeneity. Hum Mol Genet 1996;5:403-6.

11 Furukawa Y, Shimadzu M, Rajput AH, et al. GTPcyclohydrolase I gene mutations in hereditary progressive and dopa-responsive dystonia. Ann Neurol 1996;39:60917.

12 Hirano M, Tamaru Y, Ito H, et al. Mutant GTP cyclohydrolase I mRNA levels contribute to dopa-responsive dystonia onset. Ann Neurol 1997;40:796-8.

13 Lüdecke B, Dworniczak B, Bartholomé K. A point mutation in the tyrosine hydroxylase gene associated with Segawa's syndrome. Hum Genet 1995;95:123-5.

14 Matsumoto S, Takahashi R, Sadashima H, et al. Five cases of dystonia with marked diurnal fluctuation. Kitano Hosp Med 1985;30:107-10.

15 Iwami O, Kawamura J, Hashimoto S, et al. Hereditary progressive dystonia with marked diurnal fluctuation: a report of two siblings, one of them showing age-dependent changes of symptoms. Clin Neurol 1990;30:961-5.

16 Ichinose H, Ohye T, Matsuda Y, et al. Characterization of mouse and human GTP cyclohydrolase I genes. $\mathcal{F}$ Biol Chem 1995;270:10062-71.

17 Togari A, Ichinose H, Matsumoto S, et al. Multiple mRNA forms of human GTP cyclohydrolase I. Biochem Biophys Res Commun 1992;187:359-65.

18 Ringelhann B, Szelenyi JG, Horanyi M, et al. Molecular characterization of b-thalassemia in Hungary. Hum Genet characterization

19 John SWM, Rozen R, Laframboise R, et al. Novel PKU mutation on haplotype 2 in French-Canadians. Am f Hum Genet 1989;45:905-9.

20 Nar H, Huber R, Meining W, et al. Atomic structure of GTP cyclohydrolase I. Structure 1995;3:459-66.

21 Maier J, Witter K, Gütlich M, et al. Homology cloning of GTP-cyclohydrolase I from various unrelated eukaryotes by reverse-transcription polymerase chain reaction using a general set of degenerate primers. Biochem Biophys Res Commun 1995;212:705-11.

22 Witter K, Werner T, Blusch JH, et al. Cloning, sequencing and functional studies of the gene encoding human GTP cyclohydrolase I. Gene 1996;171:285-90.

23 Rajput AH, Gibb WRG, Zhong XH, et al. Dopa-responsive dystonia: pathological and biochemical observations in a case. Ann Neurol 1994;35:396-402. 